This item was submitted to Loughborough's Research Repository by the author.

Items in Figshare are protected by copyright, with all rights reserved, unless otherwise indicated.

\title{
Three modes in a radio frequency atmospheric pressure glow discharge
}

PLEASE CITE THE PUBLISHED VERSION

PUBLISHER

(C) American Institute of Physics

VERSION

VoR (Version of Record)

LICENCE

CC BY-NC-ND 4.0

REPOSITORY RECORD

Shi, J.J., Xu-Tao Deng, R. Hall, J.D. Punnett, and Michael G. Kong. 2019. "Three Modes in a Radio Frequency Atmospheric Pressure Glow Discharge". figshare. https://hdl.handle.net/2134/5171. 
This item was submitted to Loughborough's Institutional Repository (https://dspace.lboro.ac.uk/) by the author and is made available under the following Creative Commons Licence conditions.

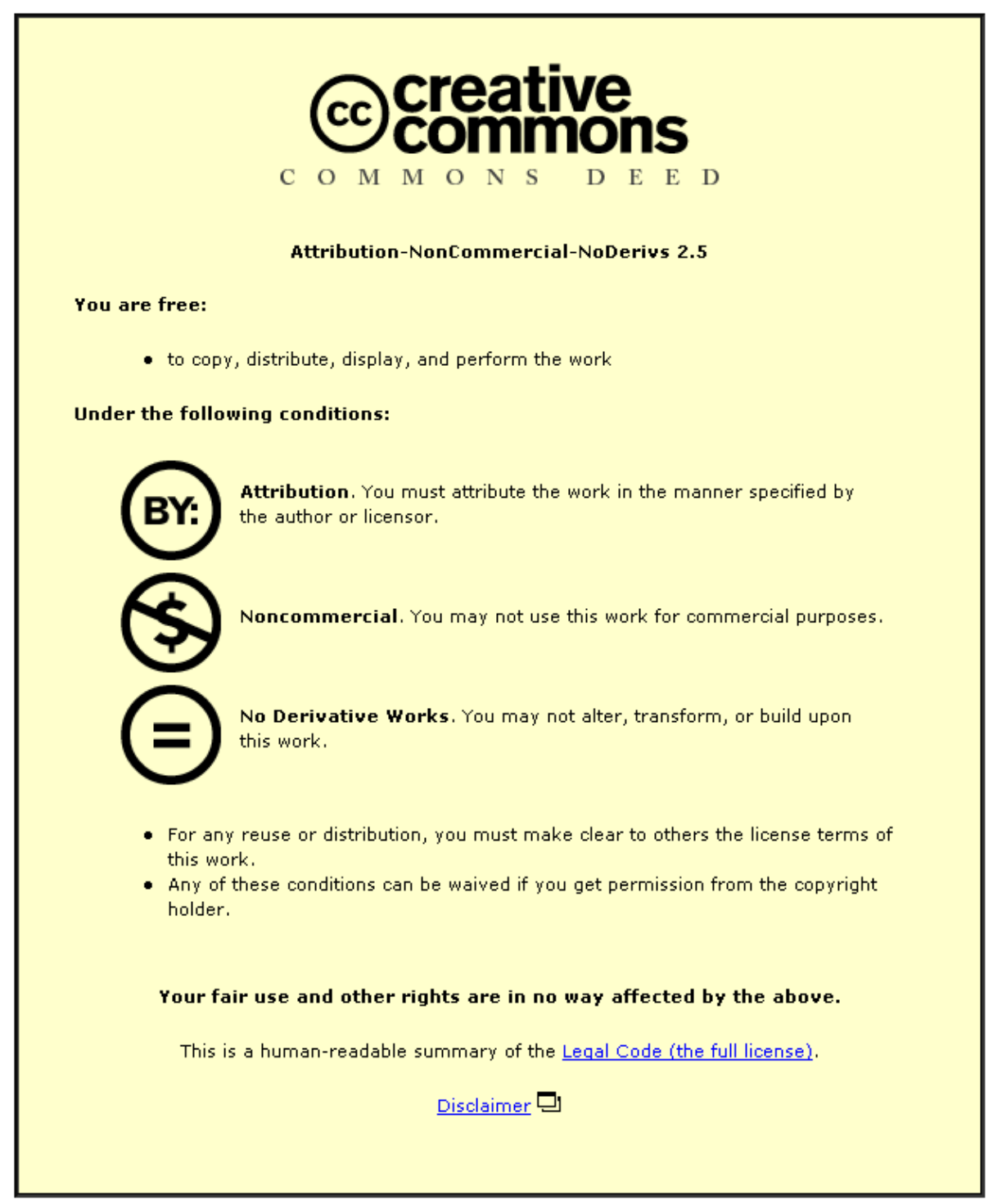

For the full text of this licence, please go to: http://creativecommons.org/licenses/by-nc-nd/2.5/ 


\title{
Three modes in a radio frequency atmospheric pressure glow discharge
}

\author{
J. J. Shi and X. T. Deng \\ Department of Electronic and Electrical Engineering, Loughborough University, Loughborough LE11 3TU, \\ United Kingdom \\ R. Hall and J. D. Punnett \\ Advanced Energy UK Ltd, Minton Place, Victoria Road, Bicester, Oxon OX6 7QB, United Kingdom \\ M. G. Konga) \\ Department of Electronic and Electrical Engineering, Loughborough University, Loughborough LE11 3TU, \\ United Kingdom
}

(Received 19 May 2003; accepted 3 September 2003)

\begin{abstract}
Fundamentally not requiring a vacuum chamber, atmospheric pressure glow discharges (APGDs) offer an exciting prospect for a wide range of material processing applications. To characterize their operation and establish their operation range, a radio frequency (rf) APGD is studied experimentally with measurement of discharge voltage, current, dissipated plasma power and plasma impedance. Different from the current understanding that rf APGD are operative only in the abnormal glow mode, we show the presence of two additional modes namely the normal glow mode and the recovery mode. It is shown that all three modes are spatially uniform and possess key characteristics of a glow discharge. So rf APGD have a much wider operation range than previously believed. To provide further insights, we investigate the transition from the abnormal glow mode to the recovery mode. It is established that the cause responsible for the mode transition is sheath breakdown, a phenomenon that is known in low- and moderate-pressure glow discharges but has not been reported before for atmospheric-pressure glow discharges. Finally we demonstrate that plasma dynamics, hence plasma stability, in these three modes are influenced crucially by the impedance matching between the plasma rig and the power source. (C) 2003 American Institute of Physics.
\end{abstract}

[DOI: $10.1063 / 1.1622110]$

\section{INTRODUCTION}

Atmospheric pressure glow discharges (APGDs) offer a vacuumless route to many material processing applications such as etching, deposition, surface modification, and sterilization. ${ }^{1-7}$ These highly collisional plasmas share some important similarities with the classical low-pressure glow discharge. For example the spatial appearance of APGD is characteristically diffuse and free of streamers, ${ }^{2}$ and their gas temperature can be reduced to near room temperature. ${ }^{7}$ The electron density of APGD lies between $10^{9}$ and $10^{13} \mathrm{~cm}^{-3}$ and their mean electron energy falls into the $1-10 \mathrm{eV}$ range. ${ }^{1,2}$ These desirable plasma properties make APGD an attractive alternative to vacuum plasmas that have been widely used in semiconductor fabrication and other material processing applications.

Atmospheric pressure glow discharges are typically capacitive plasmas generated at frequencies that span a very broad spectrum from $\mathrm{dc}^{8}{ }^{8}$ the mains frequency, ${ }^{9}$ audio frequencies $(1-100 \mathrm{kHz}),{ }^{3,5,6}$ radio frequencies $(\mathrm{rf}),{ }^{1,4}$ and microwave frequencies. ${ }^{10,11}$ APGD generated at different frequencies have their specific properties and in general it is difficult to generalize their relative merits. From a practical standpoint, $r f$ APGDs are attractive because of their low breakdown voltage. ${ }^{12}$ Over the past 3 years some of their

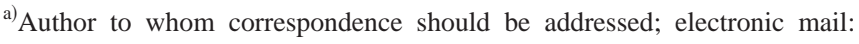
m.g.kong@lboro.ac.uk plasma properties have been investigated including emission spectrum, ${ }^{13,14}$ vibrational and rotational temperature, ${ }^{12,15,16}$ densities of excited species, ${ }^{17}$ and charged particles, ${ }^{18}$ and plasma dynamics. ${ }^{12,19}$ Yet many more aspects of their fundamental characteristics remain to be unraveled, observed and characterized before a full understanding is achieved.

One important issue is operative modes of rf APGD and the range of these modes. The current understanding is that $\mathrm{rf}$ APGD are operative only in a so-called $\alpha$ mode beyond which they evolve into high-temperature arcs. ${ }^{13}$ This has significant implications on the range and practicality of $\mathrm{rf}$ APGD since arcs are not very useful for surface modifications. In this paper we report, for the first time, experimental observation of two additional modes of rf APGD, namely the normal glow mode and the recovery mode. Our study suggests that similar to the $\alpha$ mode these two additional modes have a diffuse and uniform appearance with the ionized gas at near room temperature and that the $\alpha$ mode, hitherto considered as the only rf APGD mode, is essentially an abnormal glow mode. So rf APGD have a much wider operation range than previously believed ${ }^{12-20}$ and this greater operation range allows rf APGD to be flexibly operated for many applications. To provide further insights into these three modes, we study their mode transition mechanism also. In particular the transition from the abnormal glow mode to the recovery mode is attributed to sheath breakdown, a phenomenon that is known for low- and moderate-pressure rf glow discharges ${ }^{21}$ yet has not been observed for atmospheric pres- 


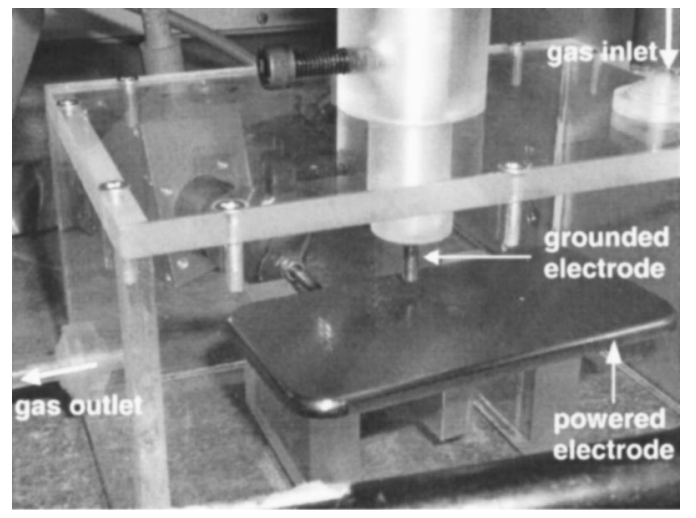

FIG. 1. Schematic of an rf atmospheric pressure glow discharge system.

sure rf glow discharges. The key contributions of this work are the first observation of the normal glow mode and the recovery mode in $\mathrm{rf}$ APGD, and the first observation of sheath breakdown in rf APGD. Our objective is to report relevant observations and then provide plausible explanation to further the current understanding. This work will form a valuable starting point for many future studies that are necessary in order to fully characterize all three modes and their mode transition mechanism. The paper is organized as follows. In Sec. II the plasma apparatus is described and main experimental data presented. Then these data are analyzed to show the presence of the three plasma modes. Section III focuses on mode transition mechanism with a simple theory of sheath breakdown. Finally key conclusions are summarized in Sec. IV.

\section{EXPERIMENTAL EVIDENCE OF THE THREE MODES}

\section{A. Experimental setup}

In our rf APGD system, the electrode unit consists of one stainless steel plate of $5 \times 10 \mathrm{~cm}^{2}$ as the powered electrode and a stainless steel rod of $4 \mathrm{~mm}$ diameter as the grounded electrode. The gap distance between the rod electrode and the plate electrode varies between 1 and $5 \mathrm{~mm}$ and is nominally $4 \mathrm{~mm}$. The rod electrode is employed to contrast impedance matching against that of a circular disk electrode. As shown in Fig. 1, the electrode unit is enclosed in a Perspex box to minimize the environmental interference and to maximize the control over the background gas. Although not airtight, this box has only two openings namely the gas inlet and the gas outlet. The working gas was helium (99.9995\% pure), fed from the gas inlet at a high flow rate. Before the rf power was switched on, the gas flow was left to persist for several minutes to ensure the majority of air originally contained within the rig was flushed out and replaced with helium. While an airtight rig may facilitate a more quantitative analysis, the rig in Fig. 1 was sufficient for the intended study of possible operative modes of $\mathrm{rf}$ APGD. The rf power was coupled to the plasma rig from a $3 \mathrm{~kW}$ rf source at $13.56 \mathrm{MHz}$ (RF30S, Advanced Energy) via an impedance matching network (AZX10, Advanced Energy). Electrical measurements were made using a rf sensor system (Z-scan, Advanced Energy), through which voltage, current,

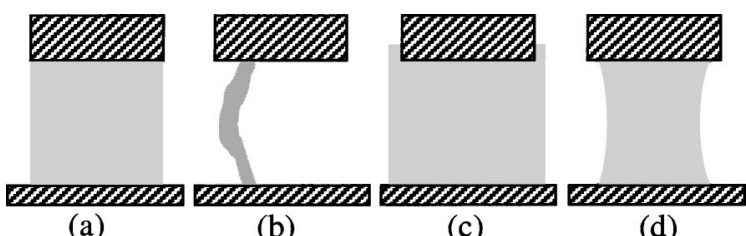

(a)

(b)

(c)

(d)

FIG. 2. Typical spatial appearance of the generated rf atmospheric glow discharge between the top rod electrode and the bottom plate electrode with (a) the normal glow mode; (b) the transition phase between the normal and abnormal mode; (c) the abnormal glow mode when the plasma encloses part of the rod electrode; and (d) the recovery mode.

current-voltage phase angle, plasma power, and plasma impedance were measured at $13.56 \mathrm{MHz}$ and its first three harmonics.

\section{B. Visual appearance of the discharge}

As the rf power was switched on and the matching impedance suitably set, a pale-purple glow was initially generated in the interelectrode gap and its visual appearance was very similar to that of the normal glow discharge generated at low pressures (e.g., milli-Torr). ${ }^{21}$ It filled the entire interelectrode space thus assuming the shape of a cylinder as shown in Fig. 2(a). It had a layered structure with a bright glow near electrodes and a comparatively dark space in the middle of the interelectrode space. This represents the normal glow mode of rf APGD. Under our experimental conditions, this pale glow was sustained with a rf power input less than $0.2 \mathrm{~W}$ or equivalently $4 \mathrm{~W} / \mathrm{cm}^{3}$. The observation of the normal glow mode has not been reported before for $\mathrm{rf}$ APGD.

With increasing rf power, the pale glow evolved into a brighter and radially shrunken plasma channel initially covering only a small area of the rod electrode surface and often moving around. This is illustrated in Fig. 2(b) and it indicates a transition from the normal glow mode to a different mode that consumed noticeably more rf power. Sometimes this transient plasma appeared first as one or two bright spots on the surface of the rod electrode without visual evidence of it connecting to the plate electrode, before an increase in $\mathrm{rf}$ power input elongated it to bridge the interelectrode gap as a moving but sustained plasma channel. As the rf power was increased further, the transient plasma channel then quickly spread the whole surface of the rod electrode and filled the entire interelectrode space so again assuming the shape of a cylinder. At this stage, the plasma was a bright white/purple glow with apparent uniformity throughout its volume. The layered structure of the normal glow mode now became blurred though there was a very thin and comparatively dark layer near the electrode surfaces. With the rf power raised still further, this bright plasma spread upwards along the rod electrode as shown in Fig. 2(c) and the plasma diameter grew from 4 to up to $8 \mathrm{~mm}$ approximately twice as large as the rod diameter. The phase from the initial thin plasma channel to the radially expanded glow represents the abnormal glow mode, also known as the $\alpha$ mode. ${ }^{13}$

When the input rf power was raised above a critical level, the abnormal glow discharge underwent an abrupt 

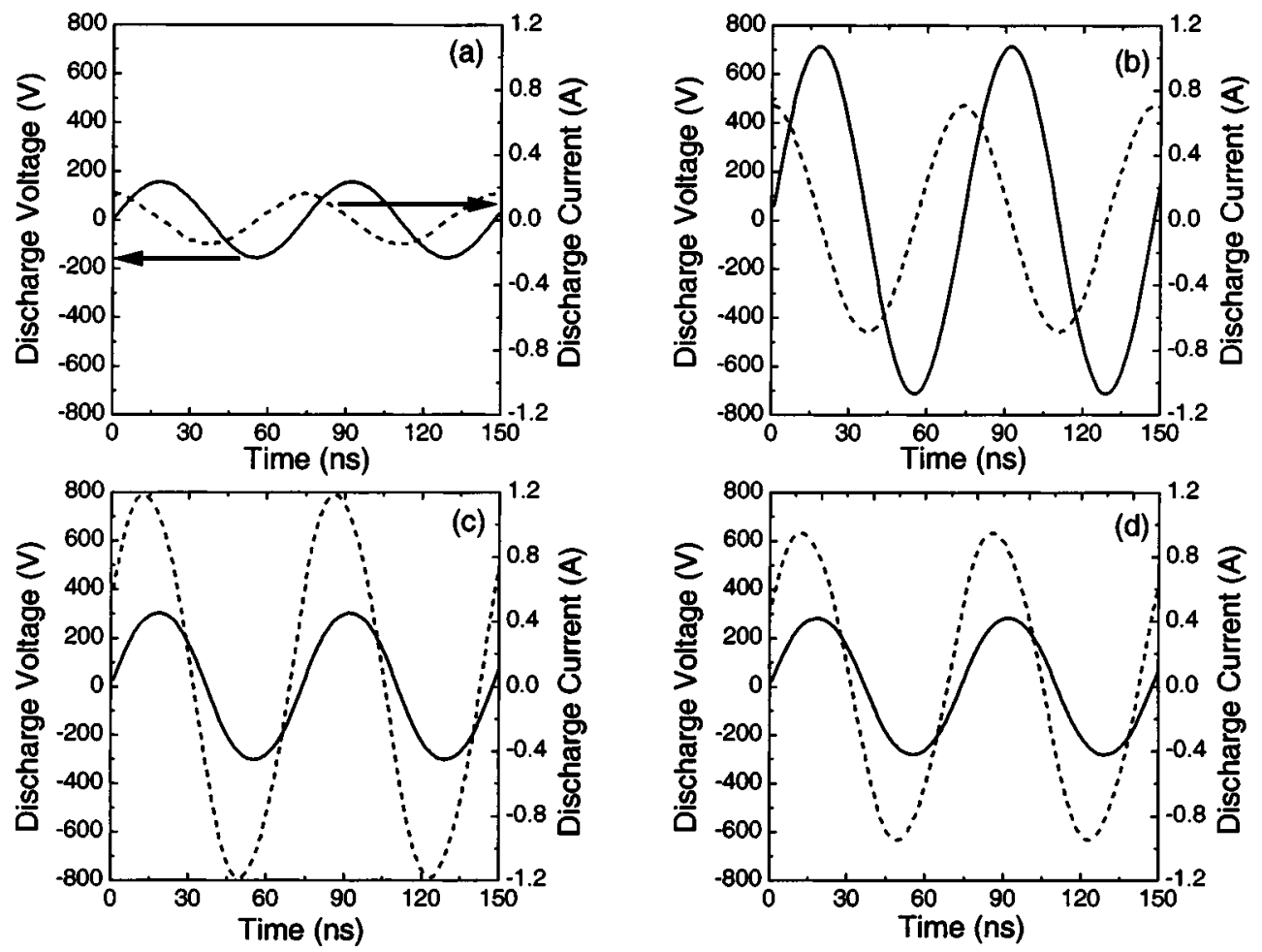

FIG. 3. Voltage (solid curve) and current (dotted curve) wave forms (a) near the beginning of the abnormal glow mode; (b) immediately before its sheath breakdown; (c) shortly after the breakdown; and (d) during recovery from the breakdown and halfway to the initial abnormal glow mode.

change and then evolved into an intensified white/orange and shrunk plasma column as illustrated in Fig. 2(d). Occasionally this abrupt change can lead to collapse and extinction of the plasma. As it will be elaborated further in the next section, this abrupt change is a result of breakdown of electrode sheath and the initially intensified plasma represents an early stage of its recovery mode following the breakdown. Importantly the discharge plasma in the recovery mode was also spatially uniform and free of streamers. With reduced power input, the diameter of the shrunken plasma cylinder increased to $4 \mathrm{~mm}$ after which it returned to its abnormal glow mode of white/purple appearance. Though the gas temperature was higher than room temperature, probably above $100^{\circ} \mathrm{C}$, a 1 min operation in the recovery mode did not leave any obvious damage mark on the surface of the bottom electrode nor heat it up markedly. It is estimated that the gas temperature in the recovery mode was no more than $150^{\circ} \mathrm{C}$ in our experiment. Given its spatial uniformity and moderate gas temperature, rf APGD in their recovery mode will be useful for uniform surface modification. This is significant as it contrasts the current understanding that the breakdown of the abnormal mode would lead to arcing and the end of the useful range of if APGD. ${ }^{13}$

\section{Voltage and current wave forms}

Figure 3 shows the voltage and current wave forms at four power input levels $(0.3,4.7,167$, and $123 \mathrm{~W})$ that, respectively, represent a point shortly after the beginning of the abnormal glow mode [Fig. 3(a)], just before [Fig. 3(b)], and shortly after the sheath breakdown [Fig. 3(c)], and at the midway in the recovery mode. These power readings have a tolerance of $<10 \%$. With a plasma volume of $0.05 \mathrm{~cm}^{3}$ they correspond to power density at $6,94,3344$, and $2443 \mathrm{~W} / \mathrm{cm}^{3}$, respectively. The power source used was rated at $3 \mathrm{~kW}$ and between 0 and $1 \mathrm{~W}$ its power output fluctuated too much for measuring reliably the current and voltage in the normal glow mode.

In all cases shown in Fig. 3, the voltage and current wave forms are predominately sinusoidal. Analysis of their frequency contents suggests that the greatest harmonic content occurred immediately before the sheath breakdown [see Fig. 3(c)] when the voltage amplitude at the third harmonic reached $3 \%$ of that at the fundamental. The current-voltage phase angle is less than $90^{\circ} \mathrm{C}$ and becomes smaller in Figs. $3(\mathrm{c})$ and 3(d), indicating that the plasma became more resistive after the breakdown. However the phase angle was always above $20^{\circ}$ and never zero. So there was always a significant capacitive element in the rf plasma generated in our experiment. These observations suggest that the rf APGD is unlikely to be arc plasma in any of its three modes since arc plasmas are more resistive and their current wave form deviates significantly from sinusoidal.

The peak discharge voltage increases from $155 \mathrm{~V}$ [Fig. 3(a)], through $714 \mathrm{~V}$ [Fig. 3(b)] and $309 \mathrm{~V}$ [Fig. 3(c)], and to 287 [Fig. 3(d)], whereas the peak discharge current changes from 0.15 A in Fig. 3(a), to 0.69 A in Fig. 3(b), 1.19 A in Fig. 3(c), and 0.96 A in Fig. 3(d). So the sheath breakdown was associated simultaneously with a voltage halving (714/ $309 \approx 2)$ and a current doubling $(1.19 / 0.69 \approx 2)$. The spaceaveraged peak electric field varies from 387 to $1785 \mathrm{~V} / \mathrm{cm}$. As the plasma diameter varies, it is more difficult to evaluate the current density. However immediately before sheath 


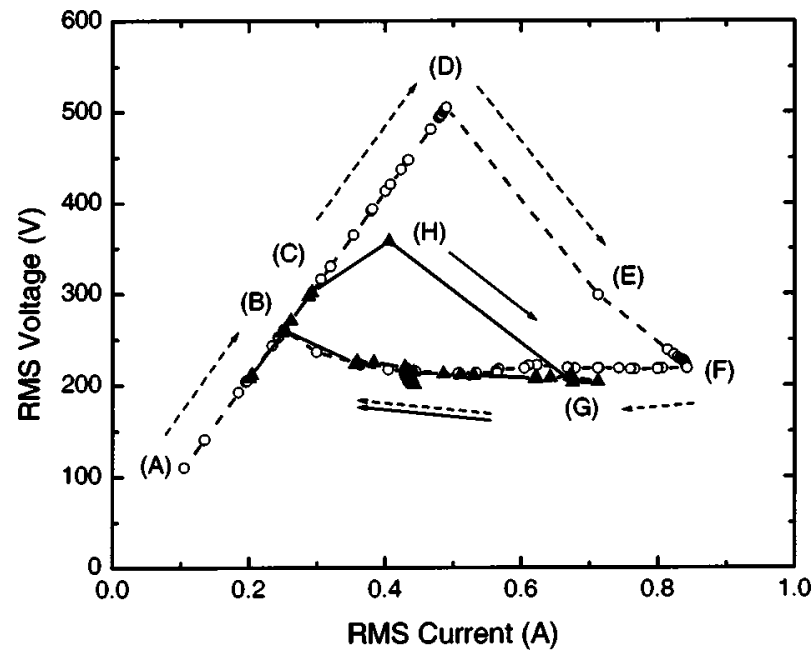

FIG. 4. Current-voltage characteristic of the generated rf plasma with the arrow showing the direction of plasma evolution. The dashed curve shows events that preceded those shown with the solid curve.

breakdown, the plasma diameter was approximately $8 \mathrm{~mm}$ suggesting a cross-sectional area of $0.50 \mathrm{~cm}^{2}$. So the peak current density immediately before sheath breakdown is 1.37 $\mathrm{A} / \mathrm{cm}^{2}$.

\section{Current-voltage characteristics}

Figure 4 shows the rms voltage as a function of the rms discharge current. The rising phase from $A$ to $D$ is very similar to that of abnormal glow discharges at low pressures ${ }^{21}$ and for this reason the discharge plasma in this phase is considered to be in the abnormal glow mode. The rising $V-I$ characteristic is also typical of that of most reported rf APGD experiments. ${ }^{12-20}$ This abnormal glow phase ended at point $D$ at which sheath breakdown was triggered and the breakdown event lasted from $D$ to $F$. During this transition period, the plasma underwent a voltage reduction of $43 \%$ and a current increment of $72 \%$. If multiple sheath breakdowns were allowed to occur in succession, via a recovery phase between two adjacent breakdowns, later breakdown events could result in current reduction rather than current increment. However as long as the experiment was performed with cold electrodes and cold background gas, current increment was always observed during sheath breakdown events. Similar breakdown observed in a comparable experiment ${ }^{13}$ was found to result in a current reduction rather than current increment observed in our experiment. This may be due to different impedance matching conditions between the rf APGD and the power source.

After the sheath breakdown and with reduced power input, the rf APGD entered into its recovery mode evolving from point $F$ to point $B$ with the gap voltage relatively unchanged in Fig. 4. Then it left its recovery mode when the current-voltage curve joined, at point $B$, the initial curve of the abnormal glow mode (e.g., $A B C D$ in Fig. 4). After the plasma returned to its abnormal glow mode and with increasing power input, it evolved briefly on the initial abnormal glow curve till point $C$, after which its $V-I$ curve departed from the initial curve and reached point $H$ when the plasma
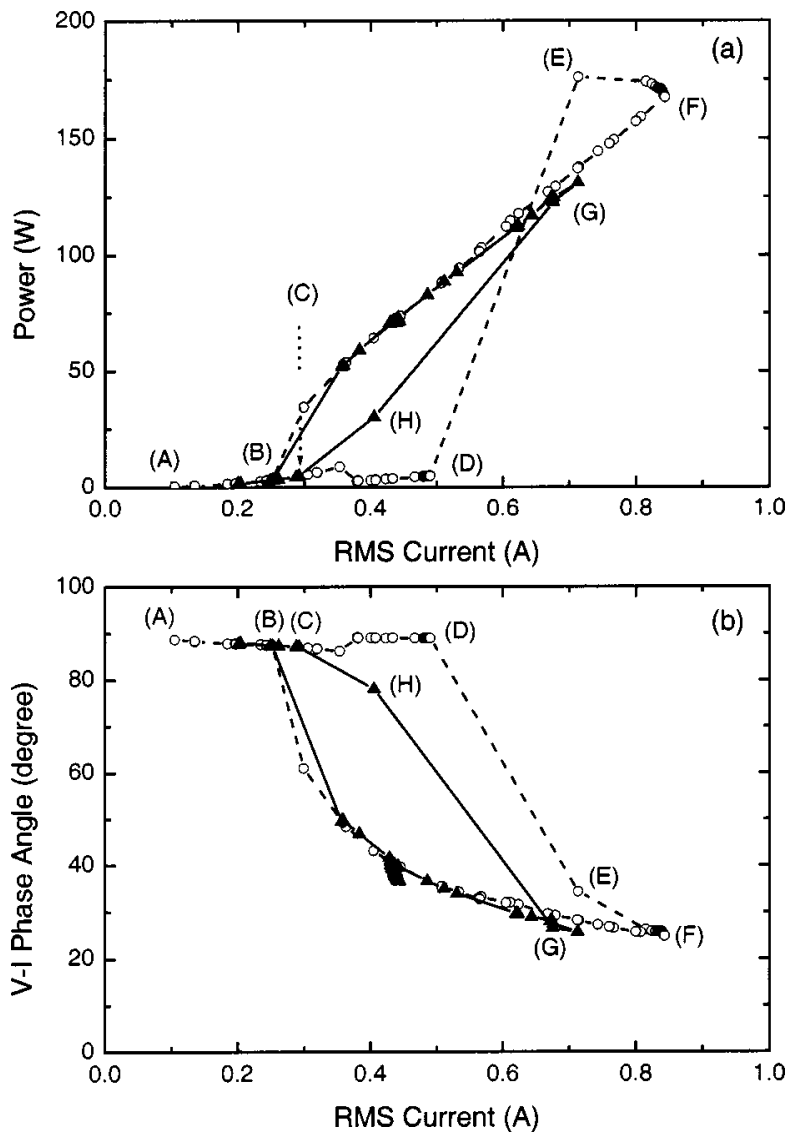

FIG. 5. Current dependence of (a) dissipated plasma power and (b) voltagecurrent phase angle. The dashed curve shows events that preceded those shown with the solid curve, and the capital letters indicate the same plasma evolution stages as those in Fig. 4.

underwent another sheath breakdown. Thus the currentvoltage characteristic shows a hysteretic behavior. Such hysteresis has not been reported before for rf APGD although it is known for glow discharges at moderate pressures (10-200 Torr). ${ }^{22}$ Subsequently from point $G$ onwards the plasma recovery from the second sheath breakdown follows roughly its recovery route from the first breakdown. Therefore the rf APGD evolves from the abnormal glow mode, through the recovery mode, then back to the abnormal glow mode. Often this evolution process automatically repeated itself with certain initial power settings. It should be mentioned that voltage and current wave forms at point $A, D, F$, and $G$ are shown, respectively, in Figs. 3(a), 3(b), 3(c), and 3(d).

\section{E. Plasma power and plasma impedance}

Plasma power and the current-voltage phase angle are shown as a function of the discharge current in Fig. 5 where points $A, B, \ldots H$ correspond to that in Fig. 4. Initially as $I_{\mathrm{rms}}$ increases from 0.1 to $0.49 \mathrm{~A}$, the plasma is in its abnormal glow mode with the power input below $5 \mathrm{~W}$. When $I_{\mathrm{rms}}$ reaches $0.49 \mathrm{~A}$, an abrupt mode transition occurs and the plasma power increases from $4.7 \mathrm{~W}$ (or $94 \mathrm{~W} / \mathrm{cm}^{3}$ ) at point $D$ to $167 \mathrm{~W}$ (or $3344 \mathrm{~W} / \mathrm{cm}^{3}$ ) at point $E$, a factor of approximately 35 . Immediately afterwards the plasma power reduces slightly to point $F$ as $I_{\text {rms }}$ increases. So the mode transition from point $D$ to point $F$ consists of initially a rapid change in 

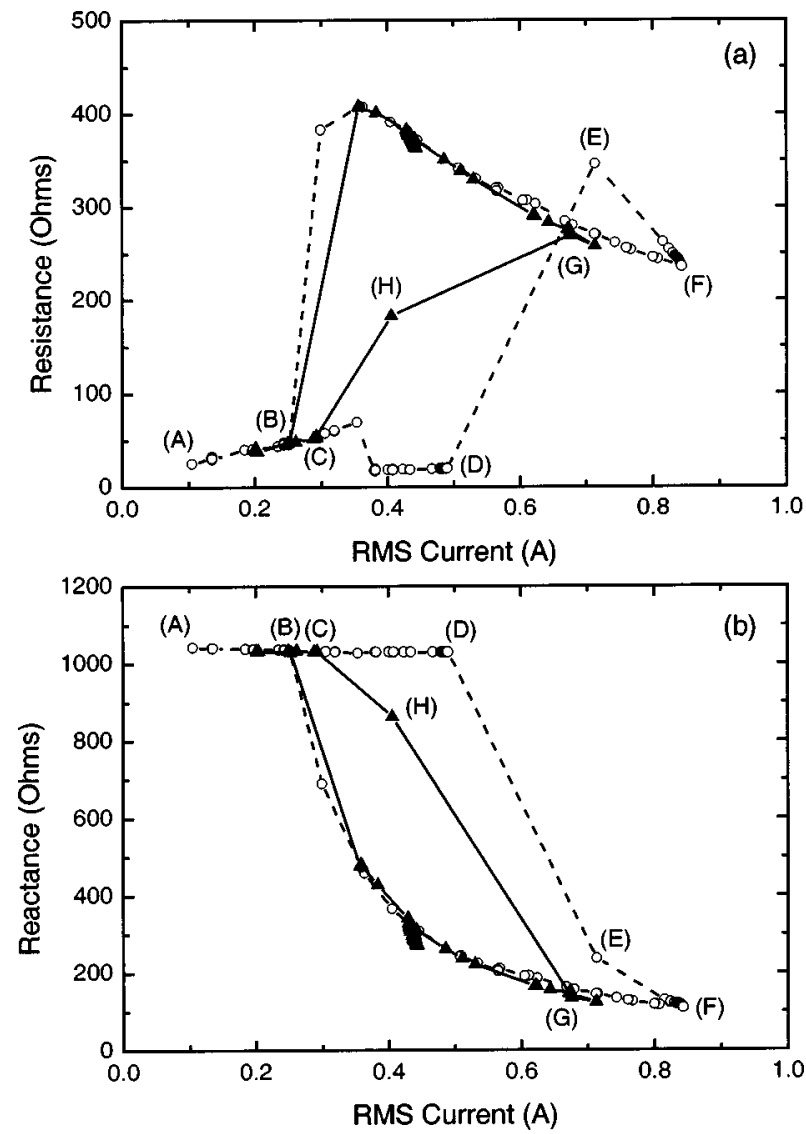

FIG. 6. Discharge current dependence of (a) plasma resistance and (b) plasma reactance. The dashed curve shows events that preceded those shown with the solid curve, and the capital letters showing plasma evolution stages are the same as those in Figs. 4 and 5.

dissipated power $(D \rightarrow E)$ and then a comparatively small adjustment $(E \rightarrow F)$. After this the plasma power reduces almost linearly as a function of current and its reduction rate is much slower than the rise rate of the power surge during sheath breakdown. This linear power reduction is typical of the recovery mode. Then a brief stay in the abnormal glow mode with a rising current leads to a second sheath breakdown at point $C$ of $I_{\mathrm{rms}}=0.29 \mathrm{~A}$ and $P=4.7 \mathrm{~W}$. The power jump of the second breakdown from point $C$ to point $G$ is $125 \mathrm{~W}$, less significant than $167-4.7 \approx 162 \mathrm{~W}$ of the first one, and $I_{\mathrm{rms}}$ reached this time is $0.68 \mathrm{~A}$ at point $G$. Interestingly point $G$ is at the same $I_{\text {rms }}$ as point $E$, both of which precede a recovery mode.

The current-voltage phase angle is shown in Fig. 5(b) and it follows a similar pattern to that of the dissipated $\mathrm{rf}$ power. The phase angle is $87^{\circ}$ at point $D$ and $25^{\circ}$ at point $F$, suggesting that the plasma evolved from being predominately capacitive to predominately resistive. To confirm this, the resistance and reactance of the plasma were measured as a function of $I_{\text {rms }}$. In Fig. 6(a), plasma resistance jumps from 20 to $347 \Omega$ before falling to $244 \Omega$ during the first sheath breakdown $(D \rightarrow F)$. During this short transition period, the plasma reactance decreases from 1029 to $117 \Omega$. So the plasma has evolved from being initially predominantly capacitive to predominantly resistive. In short, sheath breakdown is accompanied by a huge surge in dissipated power

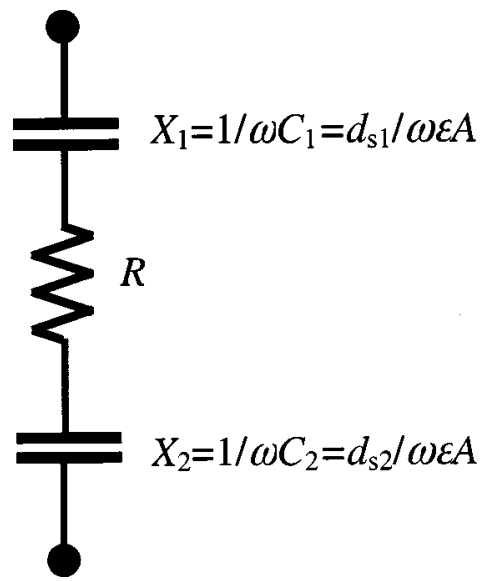

FIG. 7. A simple circuit model of rf APGD with the capacitors representing the sheaths near each of the two electrodes and the resistor representing the positive column. Both the capacitors and the resistor are functions of time to reflect plasma dynamics.

(e.g., 35 times) and plasma resistance, and a large reduction in the current-voltage phase angle and plasma reactance.

\section{MODE TRANSITION MECHANISM}

We have established that the transition from the abnormal glow mode to the recovery mode is accompanied by significant changes in key electrical parameters. For example the gas voltage is more than halved, the discharge current almost doubled, the current-voltage angle reduced from $87^{\circ}$ to $25^{\circ}$, and the dissipated $\mathrm{rf}$ power increased by 35 times. As hypothesized above, this mode transition is caused by sheath breakdown that occurs when the voltage across an electrode sheath exceeds the breakdown voltage of a gas gap of the same size as the sheath thickness. Sheath breakdown is known for low- and moderate-pressure glow discharges ${ }^{21}$ but has yet to be reported for rf APGD. Here we develop first a circuit model of rf APGD and then a sheath breakdown model.

\section{A. A circuit model of rf APGD}

The plasma may be modeled as two capacitors, each representing one of the two electrode sheaths, in series with a resistor representing the positive column that is likely to be electrically neutral and dominated by conduction current. This is illustrated in Fig. 7 and similar to the circuit model used for moderate-pressure glow discharges. ${ }^{21}$ Data of plasma reactance measurements in Fig. 6(b) are used to calculate the total capacitance. Given that the reactance $X$ $=X_{1}+X_{2}=1 / \omega C$ and $C=\epsilon A / d_{s}$, the total sheath thickness is

$$
d_{s}=d_{s 1}+d_{s 2}=\epsilon \omega A X=\pi \epsilon \omega X r_{p}^{2},
$$

where $r_{\mathrm{p}}$ is the radius of the plasma column. Although the electrode sheaths oscillate sinusoidally and sheath thickness at each electrode varies with time, the total thickness of the two electrode sheaths is constant at a given rms voltage and rms current. $^{21}$ Visual inspection of the $\mathrm{rf}$ APGD indicated $r_{p} \sim 4 \mathrm{~mm}$ before sheath breakdown occurred and at this point the plasma reactance was $1029 \Omega$. So the sheath thick- 
ness is $38.8 \mu \mathrm{m}$ according to Eq. (1). At point $F$ after the breakdown, plasma reactance is $117 \Omega$ and this leads to a reduced sheath thickness of $4.4 \mu \mathrm{m}$ at $r_{p}=4 \mathrm{~mm}$. The reduction in sheath thickness by roughly 1 order of magnitude is very similar to that in moderate-pressure glow discharges during sheath breakdown. ${ }^{21}$ This suggests strongly that sheath breakdown is responsible for the transition from the abnormal glow mode and the recovery mode.

The recovery from the sheath breakdown is associated with initially a gradual increase in plasma resistance and a faster increase in plasma reactance. The increase in plasma resistance is due to reduced plasma conductivity as the current reduction in the recovery mode relates to reduced ionization level (hence reduced electron density). On the other hand, the increase in plasma reactance is a result of a physical expansion of electrode sheath according to Eq. (1). Eventually the plasma reactance becomes $1029 \Omega$ at point $B$ and so the electrode sheath returns to the level before its breakdown. In other words the recovery phase of the plasma is characterized with electrode sheath relaxation from $d_{s}$ $=4.4 \mu \mathrm{m}$ to $d_{s}=38.8 \mu \mathrm{m}$ and a reduction in plasma conductivity.

In addition to evaluation of sheath thickness from plasma reactance data, the circuit model of rf APGD in Fig. 7 is useful for understanding relevant scaling up issues. When a disk electrode of $2 \mathrm{~cm}$ in diameter replaced the rod electrode for producing a larger volume APGD, we found that the matching network needed to be adjusted considerably before APGD can be generated. Since the disk electrode has a greater surface area, its electrode sheath has a smaller reactance $(X=1 / \omega C \sim 1 / A)$ and so the plasma rig now needs different impedance matching. Once a suitable impedance matching was achieved between the rig and the power source, stable rf APGD of larger volume was produced. With the impedance matching network used in our experiment, the largest viable disk electrode had a diameter of $3 \mathrm{~cm}$. Furthermore we found that impedance matching impacted significantly on the stability of rf APGD. For example with appropriate impedance matching and initial rf power input, it was possible for rf APGD to remain in the abnormal glow mode for more than $3 \mathrm{~h}$ continuously without experiencing any sheath breakdown. Therefore impedance matching is an important issue in scaling up rf APGD.

\section{B. A simple model of electrode sheath}

Under the influence of the applied rf power, the electrons oscillate between the two electrodes against a largely stationary ionic background and their drift towards one electrode exposes an ion-dominant region near the surface of the other electrode. This ion region is then used to model the electrode sheaths. Although this sheath model may overly simplify certain aspects of sheath behaviors, it has been used successfully to explain sheath breakdown for low- and moderatepressure rf glow discharges. ${ }^{21}$ Therefore it is used here as a first step to understand the transition from the abnormal glow mode to the recovery mode in $\mathrm{rf}$ APGD.

We assume that the applied electric field is $E(t)$ $=E_{a} \sin \omega t=\left(V_{a} / L\right) \sin \omega t$. Here $V_{a}$ is the applied rf voltage across the electrode gap, $L$ is the interelectrode gap, and $\omega$ is the radian frequency of the $\mathrm{rf}$ excitation. With the above sheath model, the rf oscillation of electrons is driven by the externally applied rf voltage and the amplitude of the rf oscillation is ${ }^{21}$

$$
x_{m}=\frac{e E_{a} / m}{\sqrt{\left(\omega^{2}-\omega_{p}^{2}\left(2 x_{m} / L\right)\right)^{2}+\omega^{2} \nu_{m}^{2}}},
$$

where $\nu_{m}=e / m \mu_{e}$ is the collision frequency and $\omega_{p}$ the plasma frequency given by

$$
\omega_{p}=\sqrt{e^{2} n_{e} / \epsilon_{0} m}=5.65 \times 10^{4} \sqrt{n_{e}\left(\mathrm{~cm}^{-3}\right)} \mathrm{s}^{-1} .
$$

Here $n_{e}$ is the electron density spatially averaged across the interelectrode gap. For helium, electron mobility $\mu_{e}=0.86$ $\times 10^{6} / p \mathrm{~cm}^{2}$ Torr/ $(\mathrm{V} \mathrm{s})^{21}$ and the gas pressure $p=760$ Torr for our experiments. So it is found that $\nu_{m}=1.55$ $\times 10^{12} \mathrm{~s}^{-1}$, much greater than $\omega=2 \pi \times 13.56 \mathrm{MHz}=8.52$ $\times 10^{7} \mathrm{~s}^{-1}$.

Immediately before the sheath breakdown at point $D$ of Figs. 4-6, the peak gap voltage was $713.9 \mathrm{~V}$, thus when spatially averaged the peak electric field was $E_{a}=713.9 / 0.4$ $=1784.8 \mathrm{~V} / \mathrm{cm}$. The breakdown of the electrode sheaths occurs when the rf oscillation of the electrons just bridges the sheath thickness, e.g., when $x_{m}=0.5 d_{s} \cdot{ }^{21}$ Under our experimental conditions, the total sheath thickness immediately before their breakdown was estimated at $d_{s}=38.8 \mu \mathrm{m}$ from plasma reactance data. Thus with $x_{m}=d_{s} / 2=19.4 \mu \mathrm{m}$ and $E_{a}=1.78 \mathrm{kV} / \mathrm{cm}$, Eqs. (2) and (3) can be used to estimate the critical electron density at which sheath breakdown occurs (e.g., point $D$ in Figs. 4-6). Assuming $\omega \ll \omega_{p}\left(2 x_{m} / L\right)^{1 / 2}$, the critical electron density is formulated as

$$
n_{\mathrm{cr}}=\frac{\epsilon_{0} m}{e^{2}} \frac{L}{2 x_{m}} \sqrt{\left(e E_{a} / m x_{m}\right)^{2}-\omega^{2} \nu_{m}^{2}} .
$$

With values relevant parameters substituted into Eq. (4), we find $n_{\mathrm{cr}}=5.21 \times 10^{13} \mathrm{~cm}^{-3}$. At $n_{e}=n_{\mathrm{cr}}, \omega_{p}=4.08 \times 10^{11} \mathrm{~s}^{-1}$ and the condition $\omega \ll \omega_{p}\left(2 x_{m} / L\right)^{1 / 2}$ is met.

In order to compare to experimental data, an additional condition has to be introduced to eliminate the electron density. To this end, we note that sheath breakdown occurs when

$$
\gamma\left(e^{\alpha d_{s}}-1\right)=1
$$

where $\alpha$ and $\gamma$ are, respectively, Townsend's first and second ionization coefficients. Although kinetic effects in electrode sheaths may be important, ${ }^{21,23}$ we employ the hydrodynamic model as an approximation and assume that the first Townsend ionization coefficient can be treated as a function of the local field only. ${ }^{5,7}$ For helium this is given by ${ }^{24}$

$$
\alpha=A_{1} p \exp \left(-B \sqrt{p / E_{s}}\right)
$$

where $A_{1}=6.5 \mathrm{~cm}^{-1}$ Torr $^{-1}$ and $B$ $=16.4 \mathrm{~V}^{1 / 2} \mathrm{~cm}^{-1 / 2} \operatorname{Torr}^{-1 / 2}$. Therefore by substituting Eq. (6) into Eq. (5), we can rewrite the breakdown condition in terms of the electric field in the electrode sheath

$$
B \sqrt{p / E_{s}}=\ln \left(A_{1} / \ln (1+1 / \gamma)\right)+\ln \left(p d_{s}\right) \equiv C_{1}+\ln \left(p d_{s}\right) .
$$




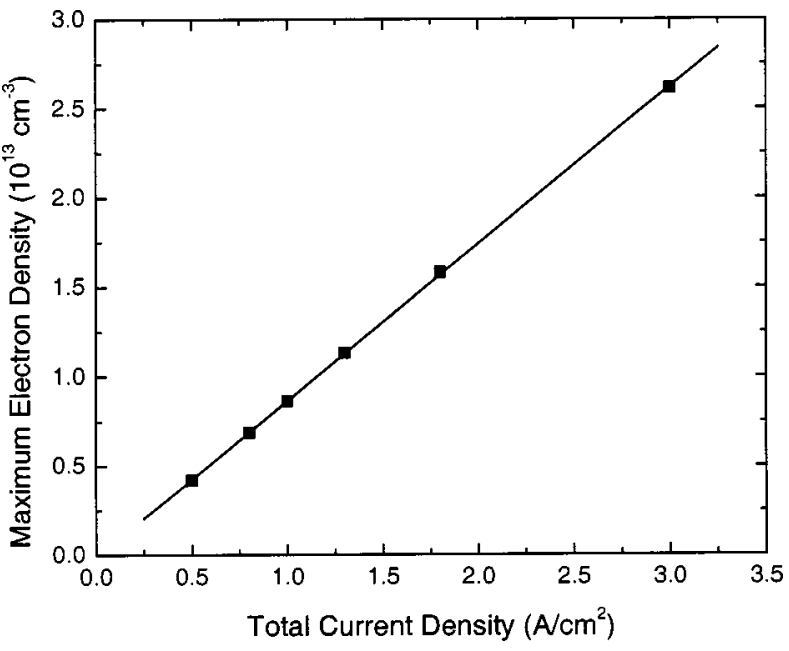

FIG. 8. Electron density as a function of the current density calculated for dc APGD (see Ref. 23) and adapted for electrode sheaths in rf APGD.

Therefore the breakdown voltage across the electrode sheath is

$$
V_{s}=E_{s} d_{s}=\frac{B^{2}\left(p d_{s}\right)}{\left(C_{1}+\ln \left(p d_{s}\right)\right)^{2}} .
$$

Sheath breakdown is triggered when the amplitude of electron oscillation reaches half of the total sheath thickness (e.g., $2 x_{m}=d_{s}$ ) and the maximum electrostatic voltage in the electrode sheaths ${ }^{21}$

$$
V_{\max }=2 e n_{e s} x_{m}^{2} / \epsilon_{0} .
$$

reaches the breakdown voltage of Eq. (8). Here $n_{\mathrm{es}}$ is the averaged electron density in the electrode sheath. It is known that electron density often peaks outside the electrode sheaths ${ }^{21,23}$ and so $n_{\mathrm{es}}$ is a fraction of $n_{c}$. According to a recent theory for dc APGD, ${ }^{23}$ typically $f_{e}=n_{\mathrm{es}} / n_{e}=0.25-1$ depending on sheath thickness. As a rough illustration of the relevant order of magnitude, we assume $f_{e}=0.5$. By equating Eqs. (8)-(9) the critical electron density immediately before the sheath breakdown (e.g., point $D$ in Figs. 4-6) can be obtained as

$$
n_{\mathrm{cr}}=\frac{\epsilon B^{2} p}{e x_{m} f_{e}\left(C_{1}+\ln \left(2 p x_{m}\right)\right)^{2}} .
$$

By substituting different values of $x_{m}$ into Eqs. (4) and (10), one can find a unique $x_{m}$ value at which the critical electron density evaluated from Eq. (4) and that from Eq. (10) becomes identical. For our experiments and at $f_{e}=0.5$ and $\gamma=0.01$, we find that this value of $\mathrm{rf}$ oscillation is $x_{m}$ $=30.85 \mu \mathrm{m}$. This should be compared to $d_{s} / 2=19.40 \mu \mathrm{m}$ extrapolated from plasma reactance data. While these two figures are within 1 order of magnitude, we note from Eq. (1) that the ratio $d_{s} / r_{p}^{2}$ is fixed at a given plasma reactance. Suppose the plasma radius was not 4 but $5 \mathrm{~mm}$, the value of rf oscillation $x_{m}=d_{s} / 2$ extrapolated from measured plasma reactance would be $30.31 \mu \mathrm{m}$, much closer to the theoretical value of $x_{m}=30.85 \mu \mathrm{m}$. Given that an error of $1 \mathrm{~mm}$ was probable in our visual determination of the plasma radius around $4 \mathrm{~mm}$, the agreement between the theoretical prediction of $x_{m}=30.85 \mu \mathrm{m}$ and the experimental data of $x_{m}$ $=19.40 \mu \mathrm{m}$ is in fact excellent. At $x_{m}=30.85 \mu \mathrm{m}$, the critical electron density is $2.06 \times 10^{13} \mathrm{~cm}^{-3}$ according to Eq. (10) and also to Eq. (4).

Given that the sheath breakdown is predominately electrostatic in nature, the cathode fall theory recently developed for dc $\mathrm{APGD}^{23}$ can be used to relate the electron density to the current density. Similar to moderate-pressure glow discharges, the electron density of APGD is approximately proportional to the current density in a linear fashion ${ }^{23}$ and Fig. 8 shows their relationship for helium according to the cathode fall theory of dc APGD. ${ }^{23}$ For $n_{e}=2.06 \times 10^{13} \mathrm{~cm}^{-3}$, the corresponding current density is $2.3 \mathrm{~A} / \mathrm{cm}^{2}$. From our earlier discussion in Sec. II C, the current density is $1.37 \mathrm{~A} / \mathrm{cm}^{2}$ immediately before sheath breakdown. This agrees favorably with the theoretical prediction of $2.3 \mathrm{~A} / \mathrm{cm}^{2}$. Therefore sheath breakdown is indeed responsible for the transition from the abnormal glow mode to the recovery mode.

\section{CONCLUSION}

rf APGD have in the past been known to be operative only in the abnormal glow mode beyond which they would evolve into arc plasmas that are much less useful for a wide range of applications. This work demonstrates the presence of two additional modes, namely the normal glow mode and the recovery mode. Experimental data are used to show that rf APGD in all three modes are spatially uniform with their gas temperature sufficient low for many surface treatment applications. So rf APGD have a much wider operation range than previously believed. In addition, the dynamic evolution of rf APGD is characterized experimentally from the abnormal glow mode, through sheath breakdown, to the recovery mode, and finally back to the abnormal mode. Under certain impedance matching conditions, this evolution process can repeat itself automatically. Yet our experiment shows that under different impedance matching conditions rf APGD can remain in the abnormal mode continually for several hours without experiencing any sheath breakdown. Impedance matching is also found to be important for scaling up rf APGD. To provide further insight into plasma dynamics evolving through the three modes, sheath breakdown is proposed as the mechanism for mode transition. A simple model of electrode sheath breakdown is developed and its prediction is found to agree well with experimental data. So sheath breakdown is indeed responsible for the transition from the abnormal glow mode to the recovery mode. These findings are important additions to the current understanding of $\mathrm{rf}$ APGD.

\footnotetext{
${ }^{1}$ A. Schutze, J. Y. Jeong, S. E. Babayan, J. Park, G. S. Selwyn, and R. F. Hicks, IEEE Trans. Plasma Sci. 26, 1685 (1998).

${ }^{2}$ E. E. Kunhardt, IEEE Trans. Plasma Sci. 28, 189 (2000).

${ }^{3}$ S. Kanazawa, M. Kogoma, T. Moriwaki, and S. Okazaki, J. Phys. D 21, 838 (1988).

${ }^{4}$ H. Koinuma, H. Ohkubo, T. Hashimoto, K. Inomata, T. Shiraishi, A. Miyanaga, and S. Hayashi, Appl. Phys. Lett. 60, 816 (1992)

${ }^{5}$ F. Massines, A. Rabehi, Ph. Decomps, R. B. Gadri, P. Segur, and C. Mayoux, J. Appl. Phys. 83, 2950 (1998).

${ }^{6}$ T. C. Montie, K. Kelly-Wintenberg, and J. R. Roth, IEEE Trans. Plasma Sci. 28, $41(2000)$
} 
${ }^{7}$ M. G. Kong and X. T. Deng, IEEE Trans. Plasma Sci. 31, 7 (2003).

${ }^{8}$ I. Alexeff et al., IEEE Trans. Plasma Sci. 30, 174 (2002).

${ }^{9}$ M. Laroussi et al., IEEE Trans. Plasma Sci. 30, 158 (2002).

${ }^{10}$ M. J. Shenton and G. C. Stevens, IEEE Trans. Plasma Sci. 30, 184 (2002).

${ }^{11}$ Y. Kabouzi, M. D. Calzada, M. Moisan, K. C. Tran, and C. Trassy, J. Appl. Phys. 91, 1008 (2002).

${ }^{12}$ J. Park, I. Henins, H. W. Hermann, and G. S. Selwyn, J. Appl. Phys. 89, 15 (2001).

${ }^{13}$ J. Park, I. Henins, H. W. Hermann, G. S. Selwyn, and R. F. Hicks, J. Appl. Phys. 89, 20 (2001).

${ }^{14}$ H. Yoshiki, A. Oki, H. Ogawa, and Y. Horiike, J. Vac. Sci. Technol. A 20, 24 (2002).

${ }^{15}$ Z. Hubicka, M. Cada, M. Sicha, A. Churpita, P. Pokorny, L. Soukup, and L. Jastrabik, Plasma Sources Sci. Technol. 11, 195 (2002).
${ }^{16}$ E. Stoffels, A. J. Flikweert, W. W. Stoffels, and G. M. W. Kroesen, Plasma Sources Sci. Technol. 11, 383 (2002).

${ }^{17}$ S. E. Babayan, G. Ding, G. R. Nowling, X. Yang, and R. F. Hicks, Plasma Chem. Plasma Process. 22, 255 (2002).

${ }^{18}$ J. Park, I. Henins, H. W. Herrmann, and G. S. Selwyn, Phys. Plasmas 7, 3141 (2000).

${ }^{19}$ X. Yuan and L. L. Raja, Appl. Phys. Lett. 81, 814 (2002).

${ }^{20}$ Y. B. Guo and F. C. N. Hong, Appl. Phys. Lett. 83, 337 (2003).

${ }^{21}$ Y. P. Raier, M. N. Shneider, and N. A. Yatsenko, Radio-Frequency Capacitive Discharges (Chemical Rubber Corp., Boca Raton, FL, 1995).

${ }^{22}$ P. Vidaud, S. M. A. Durrani, and D. R. Hall, J. Phys. D 21, 57 (1988).

${ }^{23}$ J. J. Shi and M. G. Kong J. Appl. Phys. 94, 5504 (2003).

${ }^{24}$ A. L. Ward, J. Appl. Phys. 33, 2789 (1962). 